\title{
Ossicles Autograft Safety in Cholesteatoma: A Histological Study
}

\author{
Attanasio $\mathbf{G}^{1 *}$, Mammola $\mathrm{CL}^{2}$, Covelli $\mathrm{E}^{1}$, Cagnoni $\mathrm{L}^{1}$, De Seta $\mathrm{D}^{1}$, Minni $\mathrm{A}^{1}$, Gaudio $\mathrm{E}^{2}$ and Filipo $\mathbf{R}^{1}$
}

${ }^{1}$ Department of Sensory Organs, Sapienza University of Rome, Rome, Italy

${ }^{2}$ Dept of Anatomical, Histological, Forensic and Orthopedic Sciences, Sapienza University of Rome, Rome, Italy

\begin{abstract}
Objective: The aim of the study is to define, just before any sort of cleaning procedure, if there is any epithelial inclusion inside the ossicles of patients with cholesteatoma and if the findings could be correlated with surgical aspect of cholesteatoma.

Methods: The specimens used for this study were 19 malleus and 15 incuss which were intraoperatively obtained from 24 patients. Each ossicles was grouped on the basis of cholesteatoma intraoperatively aspect as follows: Stage 1: ten ossicles obtained from encapsulated cholestatoma, non-invasive, easily cleavable. Stage 2: fourteen ossicles obtained from partially encapsulated cholesteatoma, non-invasive, non-easily cleavable. Stage 3: ten ossicles obtained from not encapsulated cholesteatoma, invasive, not cleavable. Two stapes and 1 malleus have been taken from patients who underwent middle ear surgery for conductive hearing loss and they have been used as control. The ossicles were examined histopathologically after the removal.
\end{abstract}

Results: Our results do not show any epithelial inclusion inside the ossicles independently from the macroscopic aspect or growing aggressiveness of cholesteatoma. In addition there was not inflammatory cells infiltration in stage 1 , but it was present in one incus $(7.1 \%)$ of stage 2 and in five ossicles $(50 \%)$ of stage 3 . In ossicles of grade 3 it has been found up to four layers of epithelial cells on the surface of the ossicles.

Conclusion: The results of the present study reject the hypothesis that epithelial inclusions into the ossicles could cause cholesteatoma recurrences, but strongly suggest to perform safe cleaning procedure of ossicular remnants to make them usable in ossiculoplasty in patient with partially or not encapsulated cholesteatoma.

Keywords: Cholesteatoma; Ossiculoplasty; Timpanoplasty; Incus; Malleus; Middle ear reconstruction

\section{Introduction}

Ossicular chain reconstruction or ossiculoplasty is a procedure used to re-establish the connection between the eardrum and the inner ear during middle ear surgery. Ossiculoplasty can be performed directly at the time of tympanoplasty (first-stage surgery) or after an average period of 6 months/1 year (second-stage operation). Various materials such as autologous or homologous cartilages or ossicles and synthetic prosthetic materials have been proposed to reconstruct the conductive apparatus of the middle ear. Much attention has been given to the autograft ossiculoplasty proposed for the first time by Hall and Rytzner in 1957, because of its easy retrieval, its low costs and its biocompatibility. The most commonly used autograft material is the incus body, which is often reshaped to fit between the manubrium of the malleus and the stapes capitulum. However the choice of the graft during middle ear surgery for cholesteatoma is still discussed. The main risk associated with autograft ossiculoplasty in this kind of surgery is connected to the possibility of harboring residual disease on the surface of the ossicles with a consequent failure of the entire procedure (residual cholesteatoma). For this reason alloplastic materials are the most commonly used materials for ossicular reconstruction today. With the improvement of microscopy and technologies several authors have started to try to define the best way to clean up ossicles. In 2002 Miman et al. have proposed autoclavization as a safely technique to minimize the risk of residual cholesteatoma [1]. In 2003 Siu Kwan et al. have performed a histological study to investigate whether autologous ossicles can be safely employed in ossicular chain reconstruction after attempting cholesteatoma removal with microscopic stripping or drilling [2]. With the same purpose in 2005 Ferekidis et al. assessed the status of 114 ossicles using both surgical microscope and scanning electron microscope [3]. The aim of our study is not to define the best procedure to be adopted to clean up the ossicles in autograft ossiculoplasty, but to define, just before any sort of cleaning procedure, if there is any epithelial inclusion inside the ossicles of patients with cholesteatoma and if the findings could be correlated with surgical aspect of cholesteatoma.

\section{Materials and Methods}

The specimens used for this study were 19 malleus and 15 incus which were intraoperatively obtained from 24 patients, 14 female and 10 males (mean age 56.3 years) with cholestatoma between June 2011 and July 2012. At the time of removal, all patients were operated on by the same surgeon who was asked to classify the surgical macroscopic aspects of cholesteatoma into 3 stages as follows:

Stage 1: Encapsulated cholestatoma, non-invasive, easily cleavable.

Stage 2: Partially encapsulated cholesteatoma, non-invasive, noneasily cleavable.

Stage 3: Not encapsulated cholesteatoma, invasive, not cleavable.

*Corresponding author: Giuseppe Attanasio, Department of Sensory Organs, Sapienza University of Rome, Rome, Italy, E-mail: E-mail: giuseppe.attanasio@uniroma1.it

Received September 23, 2013; Accepted November 06, 2013; Published November 08, 2013

Citation: Attanasio G, Mammola CL, Covelli E, Cagnoni L, De Seta D et al. (2013) Ossicles Autograft Safety in Cholesteatoma: A Histological Study. J Cytol Histol 4 190. doi: $10.4172 / 2157-7099.1000190$

Copyright: (c) 2013 Attanasio G, et al. This is an open-access article distributed under the terms of the Creative Commons Attribution License, which permits unrestricted use, distribution, and reproduction in any medium, provided the original author and source are credited. 
Each ossicle was assigned to one of the three grades on the basis of this classification and they were all categorized as follows:

- $\quad$ Stage 1: ten ossicles (5 malleus; 5 incus).

- $\quad$ Stage 2: fourteen ossicles (8 malleus; 6 incus).

- $\quad$ Stage 3: ten ossicles (6 malleus; 4 incus).

Two stapes and 1 malleus have been taken from patients who underwent middle ear surgery for conductive hearing loss and they have been used as control. Furthermore, it was specified whether the removed ossicle, or part of it, was in contact with the cholesteatoma or it was absorbed inside of it.

\section{Light Microscopy and Immunohistochemistry (IHC)}

Ossicles were fixed in $10 \%$ buffered formalin at room temperature for one week. Ossicles were then decalcified in 10\% EDTA Na $20.26 \%$ and $\mathrm{HCl} 2.21 \%$ solution (Decalcifying agent for histology by Carlo Erba) for three or four weeks until the specimens were pliable and soft. They were dehydrated with alcohol, cleared in xylene and embedded in paraffin, $3 \mu \mathrm{m}$ sections were stained with haematoxylin-eosin and Masson's trichrome.

Immunohistochemical (IHC) reactions were carried out according to a procedure set by the Department of Anatomical, Histological, Forensic and Orthopedic Sciences of the Pharmacology and Medicine Faculty, Sapienza University of Rome [4,5].

The approximately twenty cytokeratins -intermediate filament cytoskeletal proteins characteristic of the epithelial cells- known nowadays are classified according to their molecular weight and isoelectrical point.

Rabbit anti-Cytocheratin WSS is used to identify keratin positive epithelial cells by IHC method; it labels a variety of keratins, which constitute a wide range of molecular weight proteins. Particularly, the immunogen mainly consists of the epidermal keratin subunits 58,56 , and $52 \mathrm{kD}$ but also subunits of 60,51 and $48 \mathrm{kD}$, that is, high molecular weight cytokeratins distributed in the squamous epithelium.

For immunohistochemistry (IHC), sections were mounted on glass slides coated with $0.1 \%$ poly-L-lysine. After deparaffination, endogenous peroxidase activity was blocked by 20 -minute incubation in hydrogen peroxide $(2.5 \%)$. Sections were incubated for one hour at room temperature with antibodies for Pan-Ck (Cytocheratin Wide Spectrum Screening WSS Dako Z0622, 1:50). Samples were then rinsed with PBS for 5 minutes, incubated for 20 minutes at room temperature with secondary biotinylated antibody (Dako LSAB Plus System, HRP, Milan, Italy), then with Dako ABC (Dako LSAB Plus System, HRP) and finally developed with diaminobenzidine (Dako) to check whether the reaction took place, following the development with a microscope. In the end, the slices were counterstained with Mayer hematoxiline. For all immunoreactions, negative controls (the primary antibody was replaced with pre-immune serum) were also included.

Sections were examined in a coded fashion by Leica Microsystems DM 4500 B Light microscope). IHC observations were processed with an Image Analysis System (IAS - Delta Sistemi, Rome - Italy)

\section{Results}

In Control group, light microscopy examination showed a normal structure of bone tissue ossicles and their lamellar architecture was preserved.
In Stage I all the ossicles (five malleus and five incus) preserved the lamellar structure and no inflammatory cell infiltrate was present. No remnant of epithelial tissue was found, but two ossicles presented light features of fibrous connective tissue on the surface (Figure 1A).

One ossicle of Stage II, an incus, showed evident features of fibrous connective tissue on the surface and inflammation (Figure 1B).

In Stage III all ossicles (6 malleus; 4 incus) were preserved with their lamellar structure. In one of them, an incus, features of fibrous connective tissue on the surface and inflammation were evident. In five ossicles of this group (three malleus and two incus) lymphocytic infiltration was detected.

In Stage III part of cholesteatomatous tissue with matrix (squamous epithelium) was identified in less than $30 \%$ of ossicles that were not suitable for reconstruction. In three ossicles (three malleus), squamous epithelial tissue on the bone surface was present, but no deep invasion of metaplastic stratified squamous epithelium was observed. The perimatrix, or chorion (dermis), consisted of subepithelial connective tissue displaying inflammatory cells infiltration and limited areas of fibrous connective tissue were found in one malleus of this stade specimens. The infiltrate consisted of lymphocytes, histiocytes and plasma cells, the lymphocytes being the most predominant cell population (Figure 1C). The epithelial cell layer consisted of flat polygonal cells or cuboidal cells. The epithelium varied in thickness between one and three or four layers. The fibrous layer consisted predominantly of collagen, fibroblasts and some capillaries. The presence of iperkeratinization with anucleate squames is noted. No macrophages were seen neither in the epithelial layer nor in the fibrous layer.

Immunoistochemistry examination was negative for I and II experimental groups.

In Stage 3 Pan-CK expression was normally seen in the whole layers of squamous epithelia; the intensity of expression was the same in the suprabasal and basal layers (Figure 1D).

\section{Discussion}

Cholesteatoma is a non-neoplastic but potentially aggressive lesion characterized by the presence of keratin-producing squamous epithelium in the middle ear space or mastoid [6]. Regardless of its origin, cholesteatoma is histologically composed of keratinaceous debris in a concentric fashion surrounded by keratinizing squamous epithelium that is also called matrix $[7,8]$. The underlining subepithelial inflammatory tissue (i.e., perimatrix) consists of mononuclear and multinucleated macrophages, lymphocytes, neutrophils, fibroblasts, and endothelial cells [9]. Progressive bone erosion of the ossicles and surrounding bone is one of the hallmarks of cholesteatoma. By histology, a combination of keratinous material and stratified squamous epithelium is required to make a pathologic diagnosis of cholesteatoma. The presence of squamous epithelium in the middle ear (which is normally lined with glandular epithelium) is abnormal. Unlike the epidermis of the skin, the squamous epithelium does not contain adnexal structures or rete ridges [10].

The epithelial lining of the cholesteatoma is thinner than the dermis of the external auditory canal, missing adnexal structures, but owing all the features of the germinal layer. The subepithelial layer is 1-2 millimetres thick and is in direct contact with the underlying bone and is made of connective tissue in which fibroblasts can be found. Between the perimatrix and the bone inflammatory cells can be present. 


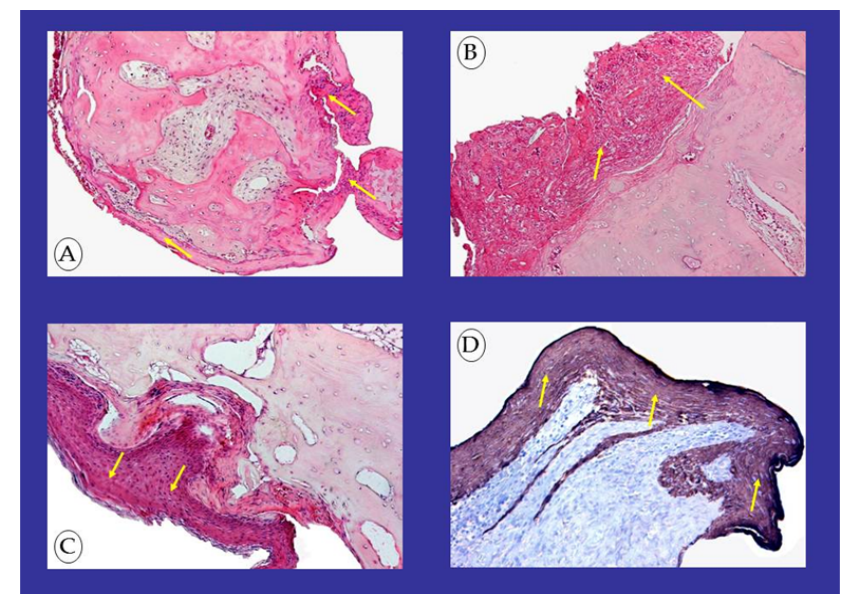

Figure 1: (A) Incus: Normal bone tissue histomorphology and regular ossicle architecture are present. Arrows: features of fibrous connective tissue on the surface. (B) An incus, showed evident features of fibrous connective tissue on the surface and inflammation (arrows). (C) Malleus head: On the surface, residual disease with squamous epithelium was observed. Inflammatory cells infiltration was present in the perimatrix. Arrows: keratinizing squamous epithelium in residual cholesteatoma; all remnant disease are superficial. (D) Malleus head: Squamous epithelia Pan-CK expression was normally seen in the whole layers of squamous epithelia. Arrows: epithelial tissue in residual cholesteatoma. (L.M., Stage I Ematoxilin-Eosin stain Original magnification 20x; L.M., Stage III Immunoistochemistry, IHC, staining to evaluate Pan-CK expression Original magnification 20x).

It often remain unclear whether cholesteatomatous epithelium is normal tissue merely in the wrong place or pathologic tissue with qualitative changes. Cytokeratins (CK) are known as the intermediate filament proteins of epithelial origin and they comprise a family of at least 19 polypeptides. Pan-CK polyclonal antibody is normally express in all epithelial tissues. The pattern of CK expression is affected only, if at all, by neoplasia and some proliferative epidermal diseases including cholesteatoma $[11,12]$. The patterns of CK expression correlate well with the state of keratinocyte proliferation, migration, and differentiation. These patterns are known to be affected during the formation of cholesteatoma.

Our results do not show any epithelial inclusion inside the ossicles independently from the macroscopic aspect or growing aggressiveness of cholesteatoma. In addition there was not inflammatory cells infiltration in grade 1, but it was present in one incus (7.1\%) of grade 2 and in five ossicles (50\%) of grade 3 . In ossicles of grade 3 it has been found up to four layers of epithelial cells on the surface of the ossicles. These observations (Stage III) are in agreement with other studies $[1,2,13,14]$.

The use of autologous ossicular remnants is considered one option in reconstruction following the eradication of cholesteatoma. However, the risk of residual cholesteatoma on the ossicular remnants may jeopardize the whole operation and predispose to recurrence and further operations. The results of the present study reject the hypothesis that epithelial inclusions into the ossicles could cause cholesteatoma recurrences, but strongly suggest to perform safe cleaning procedure as drilling, stripping or autoclaving of ossicular remnants to make them usable in ossicular chain reconstruction in patient with partially or not encapsulated cholesteatoma. We think that drilling would be better for the autograft ossiculoplasty because it allows to clean up the surface of the ossicle and at the same time it removes the inflammatory cells infiltration.

\section{References}

1. Miman MC, Aydin NE, Oncel S, Ozturan O, Erdem T (2002) Autoclaving the ossicles provides safe autografts in cholesteatoma. Auris Nasus Larynx 29: 133-139.

2. Ng SK, Yip WW, Suen M, Abdullah VJ, van Hasselt CA (2003) Autograft ossiculoplasty in cholesteatoma surgery: is it feasible? Laryngoscope 113 843-847.

3. Ferekidis E, Nikolopoulos TP, Yiotakis J, Ferekidou E, Korres S, et al. (2006) Should we use ossicular remnants in ossicular reconstruction following cholesteatoma removal? ORL J Otorhinolaryngol Relat Spec 68: 243-246.

4. Gaudio E, Barbaro B, Alvaro D, Glaser S, Francis H, et al. (2006) Vascular endothelial growth factor stimulates rat cholangiocyte proliferation via an autocrine mechanism. Gastroenterology 130: 1270-1282.

5. Gaudio E, Chaberek S, Montella A, Pannarale L, Morini S, et al. (2005) Fractal and Fourier analysis of the hepatic sinusoidal network in normal and cirrhotic rat liver. J Anat 207: 107-115.

6. Hansen T, Unger RE, Gaumann A, Hundorf I, Maurer J, et al. (2001) Expression of matrix-degrading cysteine proteinase cathepsin $\mathrm{K}$ in cholesteatoma. Mod Pathol 14: 1226-1231.

7. Chole RA (1984) Cellular and subcellular events of bone resorption in human and experimental cholesteatoma: the role of osteoclasts. Laryngoscope 94: 76 95.

8. Mills SE, Gaffey MJ, Frierson HF (2000) Tumors of the ear. Tumors of the upper aerodigestive tract and ear. 3rd series, Fascicle 26, American Registry of Pathology, Washington, DC, USA, 423-425.

9. Moriyama H, Honda Y, Huang CC, Abramson M (1987) Bone resorption in cholesteatoma: epithelial-mesenchymal cell interaction and collagenase production. Laryngoscope 97: 854-859.

10. Caponetti G, Thompson LD, Pantanowitz L (2009) Cholesteatoma. Ear Nose Throat J 88: 1196-1198.

11. Kim HJ, Tinling SP, Chole RA (2002) Expression patterns of cytokeratins in cholesteatomas: evidence of increased migration and proliferation. J Korean Med Sci 17: 381-388.

12. Kim HJ, Tinling SP, Chole RA (2002) Increased proliferation and migration of epithelium in advancing experimental cholesteatomas. Otol Neurotol 23: 840844

13. Paludetti G, Almadori G, Ottaviani F, Rosignoli M, Rossodivita M, et al (1989) [Ultrastructural aspects of cholesteatoma of the middle ear]. Acta Otorhinolaryngol Ital 9: 169-180.

14. Stankovic M (2007) Applicability of autologous incus: the influence of age and localization of cholesteatoma. Eur Arch Otorhinolaryngol 264: 995-998. 